\title{
AVALIAÇÃO DE COMPOSTOS BIOATIVOS E ATIVIDADE ANTIOXIDANTE DE EXTRATOS DE FOLHAS DE AMOREIRA PRETA (Morus nigra L.) UTILIZANDO PLANEJAMENTO EXPERIMENTAL
}

\author{
Kathlyn Schafranski ${ }^{a}$, Matheus P. Postigo ${ }^{a}$,Luciano Vitalib ${ }^{\mathrm{b}}$, Gustavo A. Micke ${ }^{\mathrm{b}}$, Wagner E. Richter ${ }^{\mathrm{a}}$ Eduardo S. \\ Chaves $^{\mathrm{b}, *, \mathbb{C}}$ \\ aDepartamento de Biotecnologia, Universidade Tecnológica Federal do Paraná, 84016-210 Ponta Grossa - PR, Brasil \\ bDepartamento de Química, Universidade Federal de Santa Catarina, 88040-970 Florianópolis - SC, Brasil
}

Recebido em 10/03/2019; aceito em 12/06/2019; publicado na web em 29/07/2019

\begin{abstract}
EVALUATION OF BIOATIVE COMPOUNDS AND ANTIOXIDANT ACTIVITY OF EXTRACTS OF BLACK MULBERRY LEAVES (Morus nigra L.) USING EXPERIMENTAL DESIGN. Extracts of black mulberry leaves present phytochemicals such as phenolic compounds and minerals. These substances have been studied because they show several health benefits. In this work we report the influence of drying temperature, infusion temperature and extraction time by means of factorial design monitoring the total phenolic content (CFT) of aqueous extracts of leaves of Morus nigra L. The concentrations of CFT, flavonoids, flavonols and antioxidant activity of the extracts were determined by spectrophotometry. The identification and quantification of phenolic compounds were performed by LC-ESI-MS / MS. The determination of the mineral concentration in the black mulberry leaves was performed by ICP-MS. The Pareto and surface response graphs suggested that the most efficient extractions occurred for leaves drying at lower temperatures and using higher infusion temperatures, extraction time did not show significant influence. The extracts presented concentration of CFT from $16.96 \pm 0.15$ to $5.73 \pm 0.33 \mathrm{mg} \mathrm{GAE} \mathrm{g}^{-1}$. The analyzes by LC-ESI-MS / MS allowed to identify phenolic substances not yet reported in the literature to date. In addition 10 mineral elements were determined in the black mulberry leaves at $\mathrm{mg} \mathrm{kg}^{-1}$ concentrations range.
\end{abstract}

Keywords: phenolic compounds; Morus nigra; factorial design.

\section{INTRODUÇÃO}

Os compostos fenólicos são uma ampla classe de bioativos, resultantes do metabolismo secundário das plantas em resposta a estresses bióticos e abióticos. ${ }^{1}$ Quimicamente, eles podem ser definidos como compostos cuja estrutura básica é um anel aromático com um ou mais substituintes hidroxila e classificados com base no número de unidades de fenol na molécula; nessa classe destacam-se os flavonoides e os ácidos fenólicos. ${ }^{2,3}$

As folhas de Morus nigra L., comumente conhecida como amoreira preta, são abundantes e possuem função importante na produção de seda, principalmente na China. São usadas na medicina tradicional, apresentam valor agronômico por servirem de biomassa para alimentação de gado, assim como alimento humano na forma de bebidas, infusões, corantes naturais e outros alimentos. ${ }^{4,5}$

Estudos com as folhas deste gênero, embora escassos, correlacionam agentes bioativos como os compostos fenólicos com diversas ações biológicas, tais como antimicrobianas, antialergênicas, efeitos antidepressivos e neuroprotetores acompanhados do decréscimo do estresse oxidativo..$^{6-8}$ Apesar das folhas de amoreira preta apresentarem altos níveis de compostos fenólicos, esses são instáveis e podem ser degradados quando submetidos a processamentos de secagem, conservação e armazenamento, principalmente quando expostos ao oxigênio e temperaturas elevadas. ${ }^{6}$

Além dos compostos bioativos presentes nas plantas e ervas medicinais, os constituintes inorgânicos também auxiliam no bom funcionamento do organismo. É, portanto, de extrema relevância que essas concentrações sejam analisadas nas folhas de amoreira preta. Elementos como o zinco, magnésio, potássio e manganês participam de muitas funções fisiológicas e bioquímicas, tais como manutenção do $\mathrm{pH}$, transmissão de impulsos nervosos, pressão

*e-mail: eduardo.chaves@ufsc.br osmótica e auxílio na regulação de diversas enzimas, podendo a carência de minerais afetar seriamente o organismo. ${ }^{9}$ Embora as folhas de Morus nigra sejam utilizadas na medicina popular em diversas regiões do mundo, estudos no que diz respeito à composição fenólica, farmacológica e avaliação das propriedades biológicas desta planta ainda são limitados.

Neste trabalho, a extração aquosa de compostos fenólicos de folhas de Morus nigra foi otimizada por meio de um planejamento experimental fatorial $2^{3} \mathrm{com}$ ponto central. As concentrações de compostos bioativos, entre estes os compostos fenólicos totais, flavonoides, flavonóis e os orto-difenólicos, além da atividade antioxidante frente ao radical DPPH'e da capacidade redutora total dos extratos obtidos foram determinadas. As classes fenólicas foram identificadas e suas concentrações nos extratos determinadas por cromatografia líquida de alta eficiência acoplada à espectrometria de massas (LC-ESI-MS/MS). Adicionalmente, a concentração total de minerais presentes nas folhas de amoreira preta foi determinada por espectrometria massa com plasma indutivamente acoplado (ICP-MS).

\section{PARTE EXPERIMENTAL}

\section{Reagentes e soluções}

Todos os reagentes utilizados foram de grau analítico. Os padrões catequina, ácidos gálico, cafeico, $p$-cumárico, ferúlico, salicílico, vanílico, siríngico, protocatecuico, quercetina e umbeliferona e demais compostos fenólicos foram obtidos na Sigma-Aldrich (St. Louis, Missouri, USA). Todos os reagentes apresentaram pureza maior do que $95 \%$. Reagentes de Folin-Ciocalteu, 2,2-difenil-1-picrilhidrazila (DPPH'), metanol, éter etílico e ácido fórmico foram obtidos na Sigma-Aldrich (São Paulo, Brasil).

Cloreto de férrico hexahidratado $\left(\mathrm{FeCl}_{3} \cdot 6 \mathrm{H}_{2} \mathrm{O}\right)$, ferricianeto de potássio $\left(\mathrm{K}_{3}\left[\mathrm{Fe}(\mathrm{CN})_{6}\right]\right)$, sulfato de cobre pentahidratado $\left(\mathrm{CuSO}_{4} \cdot 5 \mathrm{H}_{2} \mathrm{O}\right)$, 
tartarato duplo de sódio e potássio $\left(\mathrm{KNaC}_{4} \mathrm{H}_{4} \mathrm{O}_{6} \cdot 4 \mathrm{H}_{2} \mathrm{O}\right)$ e glicose anidra foram adquiridos da Alfhatec (Rio de Janeiro, Brasil). Carbonato de sódio, Cloreto de alumínio hexahidratado $\left(\mathrm{AlCl}_{3} \cdot 6 \mathrm{H}_{2} \mathrm{O}\right)$, nitrito de sódio $\left(\mathrm{NaNO}_{2}\right)$, álcool etílico, 2,3,5-trifenil cloreto de tetrazólio e isobutanol $\left(\mathrm{C}_{4} \mathrm{H}_{10} \mathrm{O}\right)$ foram adquiridos da Vetec (Rio de Janeiro, Brasil). Hidróxido de sódio $(\mathrm{NaOH})$ foi adquirido da Dinâmica (São Paulo, Brasil). Ácido clorídrico foi adquirido da Biotec (São Paulo, Brasil). Acetato de sódio anidro $\left(\mathrm{C}_{2} \mathrm{H}_{3} \mathrm{NaO}_{2}\right)$ foi adquirido da Proquimios (Rio de Janeiro, Brasil). A água ultrapura com resistividade $18,2 \mathrm{M} \Omega \mathrm{cm}$ a $25^{\circ} \mathrm{C}$ (GEHAKA Master All 2000 system, Brasil) foi utilizada no preparo das amostras e soluções. Soluções estoque $1000 \mathrm{mg} \mathrm{L}^{-1} \mathrm{em}$ metanol de cada composto fenólico foram usadas para preparação das curvas analíticas para as determinações por LC-ESI-MS/MS. Uma solução estoque multielementar ICP-3 (Perkin-Elmer) contendo $1000 \mathrm{mg} \mathrm{L}^{-1}$ dos analitos $\mathrm{Si}, \mathrm{Cu}, \mathrm{Zn}, \mathrm{V}, \mathrm{Ni}, \mathrm{Al}, \mathrm{K}, \mathrm{Mg}$ e Mn, foi utilizada para o preparo das soluções de calibrações. Peróxido de hidrogênio e o ácido nítrico foram adquiridos da Merck (São Paulo, Brasil) usados para a digestão das amostras de folhas para posterior determinação da concentração dos minerais por ICP-MS.

\section{Material vegetal}

Folhas da amoreira preta (Morus nigra L) jovens, totalmente expandidas, com pecíolo, livres de pragas e doenças e de uma mesma árvore, foram coletadas no período da manhã a uma temperatura de $18 \pm 3{ }^{\circ} \mathrm{C}$ na região de Ponta Grossa no mês de setembro, coordenadas $25^{\circ} 03^{\prime} 15.7^{\prime \prime} \mathrm{S} 50^{\circ} 08^{\prime} 02.9^{\prime \prime} \mathrm{W}$. O material coletado foi imediatamente transportado até o Laboratório de Métodos Instrumentais da Universidade Tecnológica Federal do Paraná, Campus Ponta Grossa e imediatamente higienizado. As amostras foram desidratadas em estufa de circulação de ar forçado (Fanem, 320-SE) com temperatura regulada para $50 \pm 2,75 \pm 2$ e $100 \pm 2{ }^{\circ} \mathrm{C}$ (temperaturas correspondentes, respectivamente, aos limites inferiores, ponto central e limites superiores do planejamento experimental). Em seguida, as folhas foram moídas em moinho analítico (Quimis®, São Paulo, Brasil) e peneiradas, de modo a obter uma amostra com granulometria de diâmetro inferior a $0,71 \mathrm{~mm}$. Posteriormente, estas foram armazenadas protegidas da luz, em tubos do tipo falcon escuros, e mantidas em temperatura de $-6 \pm 1{ }^{\circ} \mathrm{C}$ até o momento das análises.

\section{Planejamento experimental e preparo do extrato}

Visando simular a preparação natural de infusão de folhas de amoreira preta, a água foi selecionada como solvente extrator. Outro motivo dessa escolha é que um dos maiores desafios na extração de compostos bioativos naturais é a toxicidade alta dos solventes orgânicos e a quantidade de resíduos gerados. ${ }^{10}$

A extração dos compostos bioativos foi otimizada por meio de um planejamento experimental fatorial $2^{3}$ totalizando-se 8 experimentos com ponto central em triplicata, para investigação dos efeitos das variáveis independentes: temperatura de secagem (50, 75 e $\left.100 \pm 1{ }^{\circ} \mathrm{C}\right)$ temperatura de infusão $\left(70,83\right.$ e $\left.96 \pm 0,1^{\circ} \mathrm{C}\right)$ e tempo de extração $(10,20$ e $30 \mathrm{~min})$ sobre a variável dependente: conteúdo fenólico total.

Os extratos foram preparados utilizando-se $0,5 \mathrm{~g}$ de matéria seca, as folhas de amoreira preta em pó, para $25 \mathrm{~mL}$ de água ultrapura. As extrações foram realizadas em banho ultratermostático (Solab SL 152) com tempo e temperatura ajustados conforme o planejamento experimental. Os extratos obtidos centrifugados por 3 min a 4000 rpm (Lab 1000, Modelo DMO4125) e, em seguida, o sobrenadante foi armazenado protegido da luz, em tubos do tipo falcon escuros para posterior análise. A determinação da concentração do conteúdo fenólico total foi realizada imediatamente após a extração.

\section{Conteúdo fenólico total (CFT)}

O CFT de extratos das folhas da amoreira preta foi determinado seguindo o procedimento de Folin-Ciocalteu com algumas modificações. ${ }^{11}$ Uma alíquota de $100 \mu \mathrm{L}$ do extrato, $500 \mu \mathrm{L}$ de água e $500 \mu \mathrm{L}$ de uma solução aquosa do reagente Folin-Ciocalteau (1:3 v/v) foram adicionadas em tubo de ensaio. As amostras foram agitadas e após $5 \mathrm{~min}$ foram adicionados $500 \mu \mathrm{L}$ de $\mathrm{Na}_{2} \mathrm{CO}_{3}(10 \% \mathrm{~m} / \mathrm{v})$, mantendose em repouso por $1 \mathrm{~h}$. A absorvância da amostra foi monitorada em 720 nm utilizando espectrofotômetro UV-Vis (FEMTO 800 XI, Brasil) e cubeta de vidro com caminho óptico de $1 \mathrm{~cm}$. Uma curva de analítica foi preparada com ácido gálico $\left(R^{2}=0,9974\right)$ na faixa de concentração de 25 a $600 \mathrm{mg} \mathrm{L}^{-1}$. O experimento foi realizado em triplicata e os resultados expressos como média \pm desvio padrão em $\mathrm{mg}$ de ácido gálico equivalente por g de amostra seca (mg GAE $\left.\mathrm{g}^{-1}\right)$.

\section{Determinação de flavonoides totais}

Em uma alíquota de $250 \mu \mathrm{L}$ da amostra de extrato foram adicionados $2720 \mu \mathrm{L}$ de etanol (30\% v/v) e $120 \mu \mathrm{L}$ de uma solução de nitrito de sódio $\left(0,5 \mathrm{~mol} \mathrm{~L}^{-1}\right)$. Essas foram homogeneizadas e mantidas em repouso por $5 \mathrm{~min}$. Em seguida, adicionou-se $120 \mu \mathrm{L}$ de uma solução de cloreto de alumínio hexaidratado $\left(0,3 \mathrm{~mol} \mathrm{~L}^{-1}\right)$. Decorridos mais 5 min adicionou-se $800 \mu \mathrm{L}$ de uma solução de hidróxido de sódio (1,0 mol L $\left.{ }^{-1}\right)$ e homogeneizadas manualmente. A absorvância foi medida em $510 \mathrm{~nm}$, e a curva analítica $\left(\mathrm{R}^{2}=0,9966\right)$ foi obtida pela diluição de uma solução padrão de catequina de 10 a $100 \mathrm{mg} \mathrm{L}^{-1} \mathrm{e}$ os resultados são expressos como média \pm desvio padrão em mg de catequina equivalente por $\mathrm{g}$ de $\operatorname{amostra}$ seca $\left(\mathrm{mg} \mathrm{CAE} \mathrm{g}^{-1}\right){ }^{12}$

\section{Determinação de flavonóis totais}

O conteúdo de flavonóis foi determinado usando o método colorimétrico descrito por Yermakov et al., ${ }^{13}$ com adaptações, em que uma alíquota de $1140 \mu \mathrm{L}$ do extrato de folhas de amoreira preta diluído em água ultrapura na proporção (1:20 v/v), seguida de $1140 \mu \mathrm{L}$ de uma solução etanólica de cloreto de alumínio hexahidratado a $2 \%$ $\mathrm{m} / \mathrm{v}$ e de $1700 \mu \mathrm{L}$ de acetato de sódio $\left(0,6 \mathrm{~mol} \mathrm{~L}^{-1}\right)$. As soluções foram submetidas, por $20 \mathrm{~s}$, a agitação em vórtex e posteriormente foram mantidas em repouso, em temperatura ambiente, por 2,5 min. A absorvância foi monitorada no comprimento de onda de $420 \mathrm{~nm}$. Para quantificação dos flavonóis dos extratos uma curva analítica $\left(\mathrm{R}^{2}=0,9865\right)$ foi obtida com quercetina na faixa de concentração de 6 a $60 \mathrm{mg} \mathrm{L}^{-1}$. A análise foi realizada em triplicata e os resultados expressos como média \pm desvio padrão em $\mathrm{mg}$ de quercetina equivalente por $\mathrm{g}$ de amostra seca $\left(\mathrm{mg} \mathrm{QE} \mathrm{g}^{-1}\right)$.

\section{Sequestro de radicais livres (DPPH')}

A capacidade antioxidante foi determinada segundo método proposto por Brand-Williams et al. ${ }^{14}$ e Boroski et al. ${ }^{15}$ com algumas modificações. Os extratos da folha da amoreira preta foram pipetados em volumes crescentes $(50,100,150$ e $200 \mu \mathrm{L})$, em seguida adicionados de 3,0 mL da solução metanólica de $\operatorname{DPPH}^{*}\left(0,1192 \mathrm{mmol} \mathrm{L}^{-1}\right)$. Após 30 minutos de reação em temperatura ambiente, efetuou-se a leitura da absorvância a $517 \mathrm{~nm}$.

A capacidade de captura do radical foi determinada a partir do cálculo $\mathrm{IC}_{50}$ (Equação 1), o qual estima a concentração de antioxidante necessária para inibir $50 \%$ do radical DPPH*.

$$
\% \text { de inibição }=\left(1-\left(\frac{A b s_{517 \text { amostra }}}{A b s_{517 \text { branco }}}\right)\right) \times 100
$$


Com os resultados obtidos na Equação 1, foi obtida a curva da atividade antioxidante em função da concentração do extrato, e assim calculado por regressão linear a concentração necessária para a obtenção do $\mathrm{IC}_{50}$, ou seja, $50 \%$ da atividade antioxidante.

\section{Capacidade redutora total}

A capacidade redutora total foi analisada pelo método de Folin Ciocalteu modificado por Berker et al. ${ }^{16}$ Inicialmente, isobutanol foi utilizado para diluir o reagente de Folin na proporção de 1:2 (v/v), sendo utilizados $75 \mu \mathrm{L}$ desta solução com $50 \mu \mathrm{L}$ dos extratos das folhas de Morus nigra previamente diluídos em acetona, seguidos de $875 \mu \mathrm{L}$ de uma solução de $\mathrm{NaOH}\left(0,10 \mathrm{~mol} \mathrm{~L}^{-1}\right)$ e $1500 \mu \mathrm{L}$ de água ultrapura. A mistura resultante foi agitada em vórtex por $10 \mathrm{~s}$. Depois de 20 minutos de reação, a absorvância foi registrada $665 \mathrm{~nm}$. Quercetina foi utilizada como padrão para a obtenção da curva analítica de 72 a $360 \mathrm{mg} \mathrm{L}^{-1}\left(\mathrm{R}^{2}=0,9983\right)$. Os resultados foram expressos em $\mathrm{mg}$ de quercetina equivalente por $\mathrm{g}$ de amostra seca $\left(\mathrm{mg} \mathrm{QE} \mathrm{g} \mathrm{g}^{-1}\right.$ ).

\section{Açúcares redutores}

Os açúcares redutores possuem grupos carbonílico e cetônico livres, que detêm a capacidade de oxidar-se na presença de agentes oxidantes. Por ser um potencial interferente nas análises de fenólicos totais, faz-se necessária essa determinação. As análises foram realizadas segundo Lane e Eynon. ${ }^{17}$ A solução de Fehling foi primeiramente padronizada utilizando-se uma solução de glicose a $1 \% \mathrm{~m} / \mathrm{v}$. A partir disso, calculou-se o fator de conversão para ser usado como parâmetro nas análises das amostras.

\section{Análise de compostos fenólicos por cromatografia líquida com espectrômetro de massa (LC-ESI-MS/MS)}

Nos extratos aquosos, obtidos nas condições ótimas de extração, foram adicionados 5,0 $\mathrm{mL}$ de metanol acidificado em $\mathrm{pH}$ 2,0. Em seguida, esses foram submetidos a três ciclos de partição com éter etílico, os sobrenadantes combinados e o solvente removido por meio de um fluxo de nitrogênio. $O$ extrato seco foi ressuspenso em metanol e seus volumes aferidos para $1,0 \mathrm{~mL}$, centrifugados a $14000 \mathrm{rpm}$ por 4 minutos (Eppendorf 22331, Hamburgo Alemanha). A solução resultante foi diluída 10 vezes em solução de metanol: água (70:30 v/v) e injetada em sistema LC-ESI-MS/MS. O equipamento utilizado para as análises foi um LC system (1200 Series, Agilent Technologies, Waldbronn-BW, Germany) acoplado a um espectrômetro de massas composto por analisador triploquadrupolo e ion trap linear (Q Trap 3200 Applied Biosystems/MDS Sciex, Concord-ON, Canada). O espectrômetro foi operado com eletrospray em modo de ionização negativo.

Os compostos fenólicos foram separados em coluna Synergi ${ }^{\mathrm{TM}}$ (4.0 $\mu \mathrm{m}, 2.0$ x $150 \mathrm{~mm}$ d.i.; Phenomenex, USA). A fase móvel foi composta de uma solução (A) de metanol:água $(95: 5 \%$, v/v) e solução (B) de ácido fórmico $0,1 \%$ (v/v) em água. As condições de separação cromatográfica e do espectrômetro de massas foram exatamente as mesmas descritas por Schulz et al. ${ }^{18}$ Os compostos foram monitorados utilizando monitoramento de reações múltiplas. A identificação dos compostos foi realizada com base no tempo de retenção, íon precursor e seus fragmentos através da comparação com os respectivos padrões disponíveis comercialmente. O software Analyst ${ }^{\circledR}$ versão 1.6.2 foi utilizado para aquisição e tratamento dos dados. A quantificação foi realizada monitorando um íon quantitativo selecionado para cada composto e utilizando curvas analíticas obtidas em razão dos compostos previamente identificados com os valores da área do pico do analito versus a concentração. Os LOD (limite de detecção) e LOQ (limite de quantificação) foram obtidos a partir da relação sinal ruído de 3:1 e 10:1, respectivamente. ${ }^{19}$ As concentrações dos compostos nas amostras foram expressas como média \pm desvio padrão em $\mathrm{mg} \mathrm{g}^{-1}$ de folhas de amoreira preta.

\section{Determinação de minerais}

A concentração dos minerais foi determinada nas folhas de Morus nigra L., devidamente secas e homogeneizadas utilizando um espectrômetro de massa com plasma indutivamente acoplado (ICP-MS) modelo Elan 6000 (Perkin ElmerSciex, Thornhill, Canada). Gás argônio fornecido pela White Martins (Santa Catarina, Brasil) com pureza de $99,996 \%$ foi utilizado como gás carreador e gerador do plasma. Na Tabela 1 são descritas as condições operacionais do ICP-MS.

Para o preparo das amostras utilizou-se de $0,3 \mathrm{~g}$ de folhas secas, pesadas diretamente em frascos de politetrafluoretileno (PTFE), após a adição de 4,0 $\mathrm{mL}$ de ácido nítrico e 2,0 $\mathrm{mL}$ de peróxido de hidrogênio, e levadas à digestão em forno micro-ondas modelo DGT 100 Plus (Provecto Analítica) a 600 W. Posteriormente foram avolumados para $30 \mathrm{~mL}$ e diluídas adequadamente para análise por ICP-MS. A determinação de $\mathrm{K}$ foi realizada em fotômetro de chama modelo 610 MS (Analyser).

Tabela 1. Condições instrumentais de operação do ICP-MS

\begin{tabular}{lc}
\hline Potência de radiofrequência & $1100 \mathrm{~W}$ \\
Cone Sampler/ skimmer & $\mathrm{Pt}$ \\
Medida do Sinal & Peak Hopping \\
Unidade de medida & Área do pico \\
Resolução & $0,7 \mathrm{u}(10 \%$ altura do pico $)$ \\
Varreduras por leitura & 1 \\
Dwell time & $25 \mathrm{~ms}$ \\
Leituras por replicata & 55 \\
Replicatas & 3 \\
Vazão do gás nebulizador & $1,2 \mathrm{~L} \mathrm{~min}^{-1}$ \\
\hline
\end{tabular}

\section{Análise estatística}

A análise estatística foi realizada utilizando os softwares SasmAgri, Origin ${ }^{\circledR} 8$ e Statistica versão 10 (Statsoft, Tulsa, OK, EUA). Os resultados obtidos foram submetidos à análise de variância (ANOVA), assumindo $\mathrm{p}<0,05(5 \%)$ seguida de comparação de médias pelo teste Tukey $(\mathrm{p}<0,001)$.

\section{RESULTADOS E DISCUSSÃO}

\section{Otimização da extração por infusão}

A extração dos compostos fenólicos das folhas da amoreira preta foi realizada por meio de uma otimização multivariada onde a temperatura de secagem das folhas, temperatura e tempo de infusão foram avaliadas. As concentrações de CFT, em relação às variáveis independentes temperatura de secagem, temperatura de infusão e tempo de extração estão apresentadas na Tabela 2. Observa-se que houve diferença estatística $(p<0,001)$ entre os experimentos que compõem a matriz experimental, realizados com a mesma amostra, as concentrações de CFT obtidas variaram de 16,96 $\pm 0,15$ a $5,73 \pm 0,33 \mathrm{mg} \mathrm{GAE} \mathrm{g}^{-1}$. A literatura traz alguns estudos sobre a Morus nigra, entretanto, não foram encontrados trabalhos de avaliação dos compostos fenólicos totais após extração com água nestes moldes de 
Tabela 2. Valores médios e desvio padrão do conteúdo fenólico total de extratos aquosos de folhas de amoreira preta

\begin{tabular}{lcccc}
\hline Experimento & $\begin{array}{c}\text { Temperatura de secagem } \\
\left({ }^{\circ} \mathrm{C}\right)\end{array}$ & $\begin{array}{c}\text { Temperatura de infusão } \\
\left({ }^{\circ} \mathrm{C}\right)\end{array}$ & $\begin{array}{c}\text { Tempo } \\
(\mathrm{min})\end{array}$ & $\begin{array}{c}\text { Conteúdo Fenólico Total } \\
\left(\mathrm{mg} \mathrm{GAE} \mathrm{g}^{-1}\right)\end{array}$ \\
\hline 1 & $-1(50 \pm 1,0)$ & $-1(70 \pm 0,1)$ & $-1(10)$ & $12,59 \pm 0,54^{\mathrm{c}}$ \\
2 & $+1(100 \pm 1,0)$ & $-1(70 \pm 0,1)$ & $-1(10)$ & $5,73 \pm 0,33^{\mathrm{g}}$ \\
3 & $-1(50 \pm 1,0)$ & $+1(96 \pm 0,1)$ & $-1(10)$ & $16,61 \pm 0,53^{\mathrm{a}}$ \\
4 & $+1(100 \pm 1,0)$ & $+1(96 \pm 0,1)$ & $+1(10)$ & $8,09 \pm 0,26^{\mathrm{ef}}$ \\
5 & $-1(50 \pm 1,0)$ & $-1(70 \pm 0,1)$ & $+1(30)$ & $14,28 \pm 0,34^{\mathrm{b}}$ \\
6 & $+1(100 \pm 1,0)$ & $-1(70 \pm 0,1)$ & $+1(30)$ & $7,09 \pm 0,29^{\mathrm{f}}$ \\
7 & $-1(50 \pm 1,0)$ & $+1(96 \pm 0,1)$ & $+1(30)$ & $16,96 \pm 0,15^{\mathrm{a}}$ \\
9 & $+1(100 \pm 1,0)$ & $+1(96 \pm 0,1)$ & $0(20)$ & $8,98 \pm 0,24^{\mathrm{e}}$ \\
9
\end{tabular}

$p$-valor (ANOVA) $<0,05$; Letras distintas na última coluna indicam diferença estatística significativa ao nível de 5\% pelo teste de Tukey para os experimentos realizados.

análise. Resultados semelhantes foram reportados por outros autores que encontraram em folhas de Morus nigra coletadas na Espanha, estudo em que utilizou a extração assistida por ultrassom e metanol aquoso acidificado com éter, resultando em valores de CFT entre $13,48 \pm 0,46$ e $16,13 \pm 0,55 \mathrm{mg} \mathrm{GAE} \mathrm{g}^{-1} .^{20}$

Alguns trabalhos relatam que solventes com alta polaridade como a água podem resultar em extratos com impurezas, como ácidos orgânicos e açúcares redutores, os quais podem ter influência na determinação dos compostos fenólicos. ${ }^{21}$ Os extratos aquosos de folhas de amoreira preta apresentaram baixas concentrações de açúcares redutores $\left(0,03 \pm 0,01 \mathrm{mg} \mathrm{g}^{-1}\right)$. Dessa forma, considera-se que a influência dos açúcares redutores sobre a concentração dos compostos fenólicos não foi significativa.

Os experimentos 3 e 7 apresentaram os maiores conteúdos fenólico total, submetidos a menor temperatura de secagem $\left(50{ }^{\circ} \mathrm{C}\right)$, maior temperatura de infusão $\left(96^{\circ} \mathrm{C}\right)$ e tempos de infusão de 10 e 30 minutos respectivamente. Com o Gráfico de Pareto (Figura 1) é possível uma melhor compreensão visual do comportamento do CFT em relação às variáveis independentes e suas interações. A linha em vermelho indica a região onde as variáveis devem alcançar para demonstrar significância estatística.

Pode se observar que a temperatura de secagem, temperatura de infusão e tempo foram significativas para o conteúdo fenólico total, assim como as interações entre a temperatura de secagem das folhas e a temperatura de infusão, como também entre temperatura de infusão e o tempo de extração. A Tabela 3 fornece resultados sobre os efeitos das variáveis e os coeficientes de regressão e assim é possível verificar se os fatores (variáveis independentes) foram significativos.

$\mathrm{O} p$-valor pode variar de 0 a 1 e simboliza a probabilidade ou chance do efeito observado pelas variáveis ser devido aos fatores que

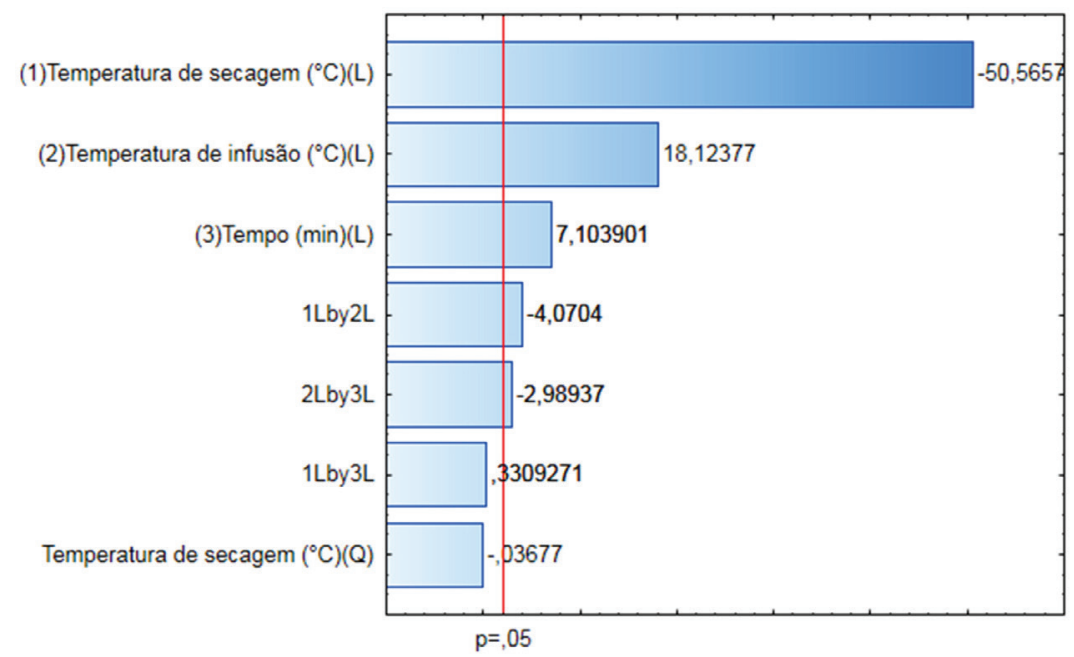

Figura 1. Gráfico de Pareto - Efeito da temperatura de secagem, temperatura de infusão e tempo no conteúdo fenólico total de folhas de amoreira preta

Tabela 3. Estimativa dos efeitos de fatores selecionados no conteúdo fenólico total para extratos de folhas de amoreira preta

\begin{tabular}{lcccccc}
\hline Fatores & Efeito & Erro padrão & t calc & $p$-valor & $\begin{array}{c}\text { Limite de confiança } \\
(-95 \%)\end{array}$ & $\begin{array}{c}\text { Limite de confiança } \\
(+95 \%)\end{array}$ \\
\hline Temperatura de secagem $\left({ }^{\circ} \mathrm{C}\right)$ & $-7,6400$ & 0,1511 & $-50,5657$ & 0,0000 & $-7,9574$ & $-7,3226$ \\
Temperatura de infusão $\left({ }^{\circ} \mathrm{C}\right)$ & 2,7383 & 0,1511 & 18,1238 & 0,0000 & 2,4209 & 3,0558 \\
Tempo (min) & 1,0733 & 0,1511 & 7,1039 & 0,0000 & 0,7559 & 1,3908 \\
Interação temperaturas de secagem e infusão $\left({ }^{\circ} \mathrm{C}\right)$ & 0,6150 & 0,1510 & 4,0704 & 0,0007 & 0,9324 & 0,2975 \\
\hline
\end{tabular}


estão sendo investigados, e não aos erros randômicos que causam flutuações normais nos resultados. Para que os efeitos observados sejam significativos estatisticamente, o $p$-valor deve ser menor ou igual a 0,05 , assumindo como margem de segurança $5 \%$ de chances de erro. ${ }^{22}$ Portanto, estatisticamente para o método do conteúdo fenólico total, o modelo experimental, a curvatura e todas as variáveis independentes (temperatura de secagem, temperatura de infusão e tempo) foram significativas.

A concentração de compostos fenólicos presentes teve uma queda média de $8 \%$ na secagem das folhas em temperatura de $100{ }^{\circ} \mathrm{C} \mathrm{em}$ relação à temperatura de $50{ }^{\circ} \mathrm{C}$. Ao utilizar temperatura de infusão a $96{ }^{\circ} \mathrm{C}$ o CFT aumentou em média $3 \%$ do que em temperatura de $70{ }^{\circ} \mathrm{C}$. Os efeitos da temperatura de secagem sobre a estabilidade de compostos fenólicos também foram investigados por Larrauri et al., ${ }^{23}$ ao analisarem o CFT em cascas de bagaço de uva vermelha, observaram que a secagem em estufa a $60{ }^{\circ} \mathrm{C}$ não afetou a estabilidade, mas houve uma redução considerável do CFT quando secas a $100{ }^{\circ} \mathrm{C}$. Esta análise pode ser confirmada pelo gráfico de superfície de resposta (Figura 2) o qual avalia a influência de duas variáveis independentes em relação a resposta desejada.

Analisando as superfícies de resposta é possível observar que todos os gráficos apresentaram inclinação ascendente, as regiões ótimas de extração estão localizadas onde se obtém as maiores concentrações de CFT. Como não foi observada uma curvatura significativa, procura-se a condição em direção da região ótima de extração. A realização de novos experimentos, deslocando os experimentos em direção à condição ótima não é possível, já que a temperatura máxima de extração é limitada pelo banho ultratermostático e além disso o objetivo deste estudo é simular o preparo natural e caseiro de uma infusão das folhas de amoreira preta.

Conforme pode ser observado, a temperatura de secagem foi a variável independente (fator) que obteve maior significância dentre os três fatores analisados, sendo que a sua significância é atribuída à condição -1, que se refere à utilização da temperatura de secagem das folhas a $50{ }^{\circ} \mathrm{C}$. A temperatura de infusão foi a segunda variável independente (fator) que obteve maior efeito e a sua significância é atribuída à condição +1 , demonstrando que é mais significativo utilizar uma temperatura de infusão a $96{ }^{\circ} \mathrm{C}$ do que $70{ }^{\circ} \mathrm{C}$, que no caso deste estudo, foi a matriz que apresentou maior percentual de conteúdo fenólico total. Embora o tempo tenha se mostrado significativo estatisticamente, seu efeito é muito menos pronunciado do que o efeito dos dois outros fatores principais, sendo que um menor tempo implicaria em menor gasto de energia.

Recentemente, Casagrande et al. ${ }^{24}$ ao investigarem a influência da temperatura de extração no conteúdo fenólico total em alecrim do campo, observaram que ao aumentar a temperatura de extração de 40
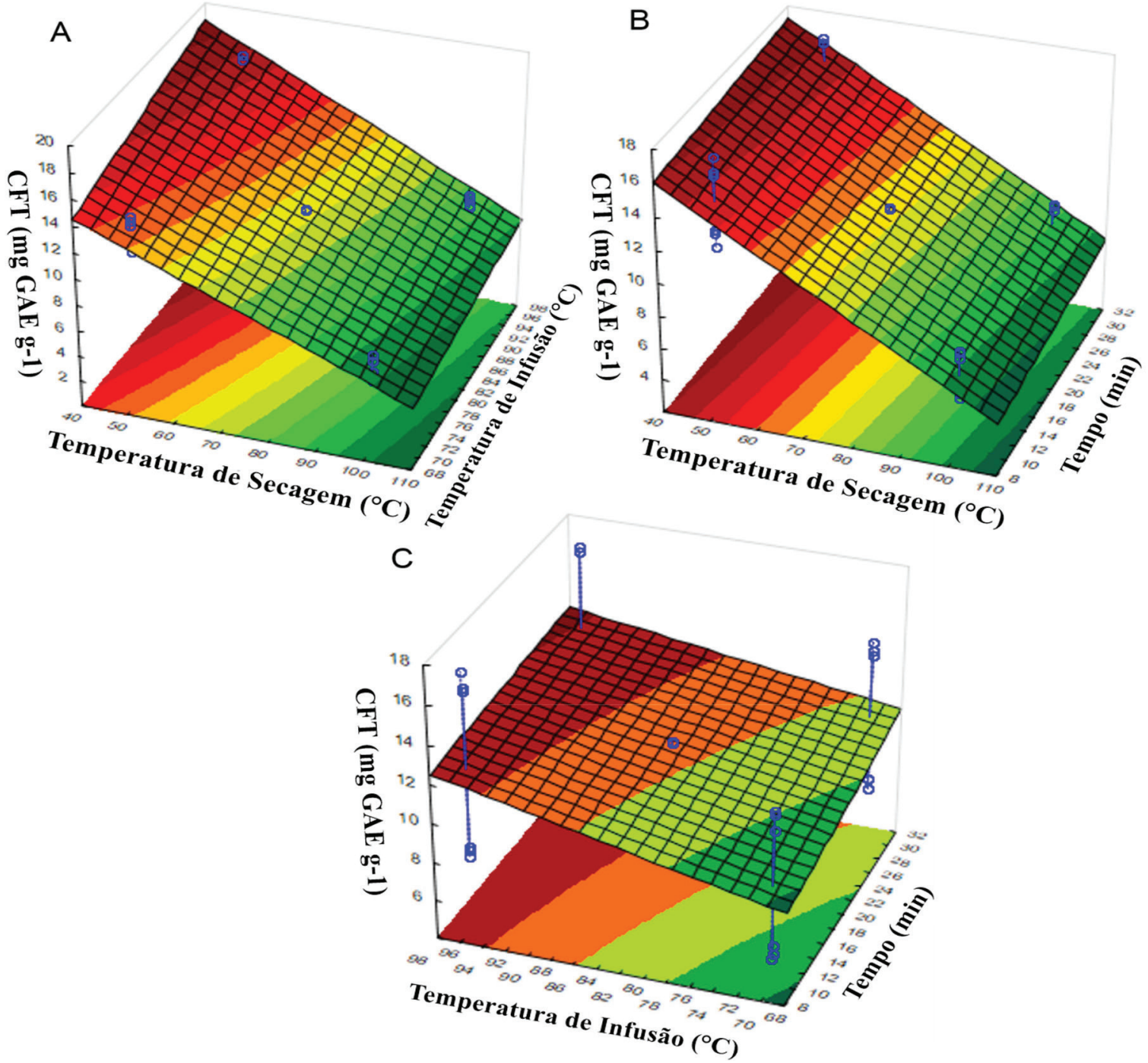

Figura 2. Gráficos de superfície de resposta para conteúdo fenólico total em função de: Temperatura de secagem versus Temperatura de infusão (A); Temperatura de secagem versus Tempo (B); Temperatura de infusão versus Tempo $(C)$ 
para $80{ }^{\circ} \mathrm{C}$ resultou em um aumento de $50 \%$ no CFT. Rajha et al.,$^{25}$ por meio de um planejamento fatorial para otimizar a extração etanólica em amostras de uvas, alcançaram maior quantificação de CFT a temperatura de extração de $94{ }^{\circ} \mathrm{C}$. As condições ótimas de extração dos CFT foram: temperatura de secagem a $50{ }^{\circ} \mathrm{C}$, temperatura de infusão a $96{ }^{\circ} \mathrm{C}$ por um tempo de 10 minutos. Estas condições foram adotadas para obtenção dos extratos e para posterior análise.

\section{Composição química e atividade antioxidante dos extratos de amoreira preta}

Para os extratos obtidos nas condições ótimas, as concentrações de flavonoides totais, flavonóis totais, capacidade redutora total e capacidade antioxidante foram determinadas. Os resultados obtidos indicam que as concentrações de flavonoides totais foram de 4,01 $\pm 0,16 \mathrm{mg} \mathrm{CAE} \mathrm{g}^{-1}$, sendo esses semelhantes aos relatados na literatura. Thabti et al..$^{26}$ constataram concentrações de flavonoides totais de 1,93 a 3,98 $\mathrm{mg} \mathrm{RE} \mathrm{g}^{-1}$ (Equivalente de Rutina) em folhas de diferentes espécies de amoreira com extração água/metanol $(1: 1, \mathrm{v} / \mathrm{v})$ em banho maria a $80^{\circ} \mathrm{C}$ por 15 minutos. A concentração de flavonóis totais nos extratos de folha de amoreira preta foi de $2,67 \pm 0,13 \mathrm{mg} \mathrm{QE} \mathrm{g}^{-1}$. Até agora, nenhuma espécie de amoreira preta teve dados reportados para a concentração total de favonóis. Os valores encontrados para a capacidade redutora total e $\mathrm{IC}_{50}$ foram de 27,69 \pm 0,46 $\mathrm{mg} \mathrm{QE} \mathrm{g}^{-1}$ e 0,94 $\pm 0,12 \mathrm{mg} \mathrm{mL}^{-1}$ respectivamente. Resultados semelhantes foram registrados por uma pesquisa em que se observaram valores de atividade antioxidante em extratos aquosos de folhas de amoreira preta de $\mathrm{IC}_{50}$ de $1,25 \mathrm{mg} \mathrm{mL}^{-1} \cdot{ }^{27}$ Essa capacidade antioxidante em extratos aquosos de folhas de Morus nigra tem sido demonstrada em estudos in vivo. Um estudo verificou os efeitos causados por uma dose de $400 \mathrm{mg} \mathrm{kg}^{-1}$ dia $^{-1}$ ofertada a ratas Wistar grávidas diabéticas e não diabéticas. Além de apresentarem redução nas taxas de colesterol total, colesterol LDL (low density lipoproteins) e triglicerídeos, foi observado também que a oferta do extrato aquoso elevou os níveis sanguíneos da enzima superóxido dismutase, demonstrando que os extratos aquosos das folhas de amoreira preta apresentam ação antioxidante. ${ }^{28}$

\section{Análise de compostos fenólicos de extratos por LC-ESI-MS/MS}

\section{Parâmetros de desempenho analítico para análise}

A faixa linear, o coeficiente de determinação $\left(\mathrm{R}^{2}\right)$, os limites de detecção (LOD) e quantificação (LOQ) e desvio padrão relativo
(RSD) obtido para a determinação dos compostos fenólicos por LCESI-MS/MS são apresentados na Tabela 4.

Coeficientes de determinação maiores do que 0,99 foram obtidos, exceto para o padrão ácido vanílico, indicando boas correlações entre as concentrações dos compostos investigados e as áreas das bandas, no intervalo de concentração da curva analítica. O limite de detecção é definido como a menor concentração do composto que está sendo analisado e que pode ser detectado, mas não necessariamente quantificado pelo método experimental, já o limite de quantificação é caracterizado como a menor concentração do composto sob as condições experimentais estabelecidas que pode ser quantificado. ${ }^{19} \mathrm{~A}$ precisão do método proposto foi avaliada por meio do desvio padrão relativo (RSD), o qual apresentou resultados entre $0,8 \%$ a $9,2 \%$, demonstrando uma boa precisão.

\section{Perfil fenólico dos extratos de amoreira preta}

Os compostos fenólicos presentes nos extratos de folhas amoreira preta também foram identificados e quantificados por LC-ESI-MS/MS. As concentrações dos compostos, os íons precursores juntamente com o tempo de retenção estão descritos na Tabela 5 e cromatogramas Figura 3.

A quercetina (10) foi encontrada no extrato de folhas de amoreira preta em uma concentração aproximada de $2,429 \mathrm{mg} \mathrm{g}^{-1}$ por peso seco da amostra. Alguns autores identificaram a quercetina como um dos componentes mais abundantes em folhas de amoreira. Pesquisas com camundongos evidenciaram que o consumo dietético de folhas de amoreira atenuou o desenvolvimento de lesões ateroscleróticas e esses efeitos foram atribuídos a quercetina. ${ }^{6,29} \mathrm{O}$ ácido protocatecuico apresentou concentração de 1,247 $\pm 0,068 \mathrm{mg} \mathrm{g}^{-1}$. Um estudo com extratos de folhas de Morus nigra e Morus alba obtidos por maceração e fluído supercrítico demonstrou concentrações de ácido protocatecuico entre 0,4365 e $0,7369 \mathrm{mg} \mathrm{g}^{-1} .^{30}$

Conforme a Tabela 6, o ácido cafeico (4) apresentou-se em concentração significativa no extrato de folhas de amoreira preta com $0,142 \pm 0,015 \mathrm{mg} \mathrm{g}^{-1}$. Thabti et al. ${ }^{26} \mathrm{em}$ suas pesquisas com extratos metanólicos de folhas de amoreira branca, constataram que o ácido cafeico era majoritário $\left(15,841 \mathrm{mg} \mathrm{g}^{-1}\right)$.

Outros derivados do ácido benzóico foram encontrados nos extratos foliares da amoreira preta, como os ácidos vanílico, siríngico, 4-hidroximetilbenzoico, salicílico e elágico em menores concentrações, além dos ácidos p-cumárico e clorogênico, que são derivados do ácido cinâmico. Os ácidos clorogênico, vanílico, siríngico e $p$-cumárico já haviam sido relatados na literatura como constituintes em folhas de

Tabela 4. Parâmetros de desempenho analítico para determinação de compostos fenólicos por LC-ESI-MS/MS

\begin{tabular}{|c|c|c|c|c|c|}
\hline Composto & Faixa linear $\left(\mathrm{mg} \mathrm{L}^{-1}\right)$ & $\mathrm{R}^{2}$ & $\operatorname{LOD}\left(\mathrm{mg} \mathrm{L}^{-1}\right)$ & LOQ $\left(\mathrm{mg} \mathrm{L}^{-1}\right)$ & $\%, \mathrm{RSD}$ \\
\hline Ácido Protocatecuico & $0,08-1,00$ & 0,9957 & 0,02 & 0,07 & 1,0 \\
\hline Ácido 4-hidroximetilbenzóico & $0,05-6,18$ & 0,9978 & 0,001 & 0,003 & 2,7 \\
\hline Ácido clorogênico & $0,03-2,36$ & 0,9952 & 0,003 & 0,01 & 7,6 \\
\hline Ácido cafeico & $0,05-4,00$ & 0,9917 & 0,02 & 0,05 & 4,6 \\
\hline Ácido vanílico & $0,40-3,00$ & 0,9852 & 0,01 & 0,02 & 5,3 \\
\hline Ácido siríngico & $0,08-6,00$ & 0,9958 & 0,001 & 0,002 & 1,1 \\
\hline Ácido $p$-cumárico & $0,31-4,08$ & 0,9937 & 0,001 & 0,01 & 1,8 \\
\hline Ácido ferúlico & $0,06-0,61$ & 0,9946 & 0,01 & 0,03 & 9,7 \\
\hline Umbeliferona & $0,08-6,06$ & 0,9903 & 0,01 & 0,04 & 9,2 \\
\hline Quercetina & $0,08-3,21$ & 0,9973 & 0,01 & 0,04 & 0,8 \\
\hline Ácido salicílico & $0,10-4,00$ & 0,9955 & 0,004 & 0,01 & 1,6 \\
\hline Ácido elágico & $0,05-6,00$ & 0,9995 & 0,01 & 0,02 & 1,6 \\
\hline
\end{tabular}


Tabela 5. Concentração dos compostos fenólicos (média \pm desvio padrão) determinada e por LC-ESI-MS/MS em extratos de folhas de amoreira preta

\begin{tabular}{|c|c|c|c|c|c|}
\hline Sinal & Composto & Concentração $\left(\mathrm{mg} \mathrm{g}^{-1}\right)$ & $\begin{array}{c}\text { Íon precursor }(\mathrm{m} / \mathrm{z}) \\
\mathrm{Q} 1\end{array}$ & $\begin{array}{c}\text { Íon quantitativo }(\mathrm{m} / \mathrm{z}) \\
\text { Q3 }\end{array}$ & $\begin{array}{l}\text { Tempo de retenção } \\
\text { (min) }\end{array}$ \\
\hline 1 & Ácido protocatecuico & $1,247 \pm 0,068$ & 136,9 & 109,0 & 6,95 \\
\hline 2 & Ácido 4-hidroximetilbenzóico & $0,062 \pm 0,003$ & 150,8 & 104,2 & 8,84 \\
\hline 3 & Ácido clorogênico & $0,029 \pm 0,003$ & 352,8 & 187,8 & 9,19 \\
\hline 4 & Ácido cafeico & $0,142 \pm 0,015$ & 178,8 & 131,3 & 9,45 \\
\hline 5 & Ácido vanílico & $0,030 \pm 0,002$ & 166,8 & 148,5 & 9,65 \\
\hline 6 & Ácido siríngico & $0,007 \pm 0,001$ & 196,8 & 119,6 & 10,01 \\
\hline 7 & Ácido $p$-cumárico & $0,004 \pm 0,001$ & 163,0 & 119,0 & 10,46 \\
\hline 8 & Ácido ferúlico & $0,096 \pm 0,004$ & 192,8 & 129,7 & 10,73 \\
\hline 9 & Umbeliferona & $0,261 \pm 0,009$ & 160,8 & 129,5 & 10,78 \\
\hline 10 & Quercetina & $2,429 \pm 0,538$ & 301,0 & 149,3 & 10,84 \\
\hline 11 & Ácido salicílico & $0,028 \pm 0,002$ & 136,9 & 91,1 & 10,99 \\
\hline 12 & Ácido elágico & $0,045 \pm 0,001$ & 300,8 & 142,5 & 11,71 \\
\hline
\end{tabular}

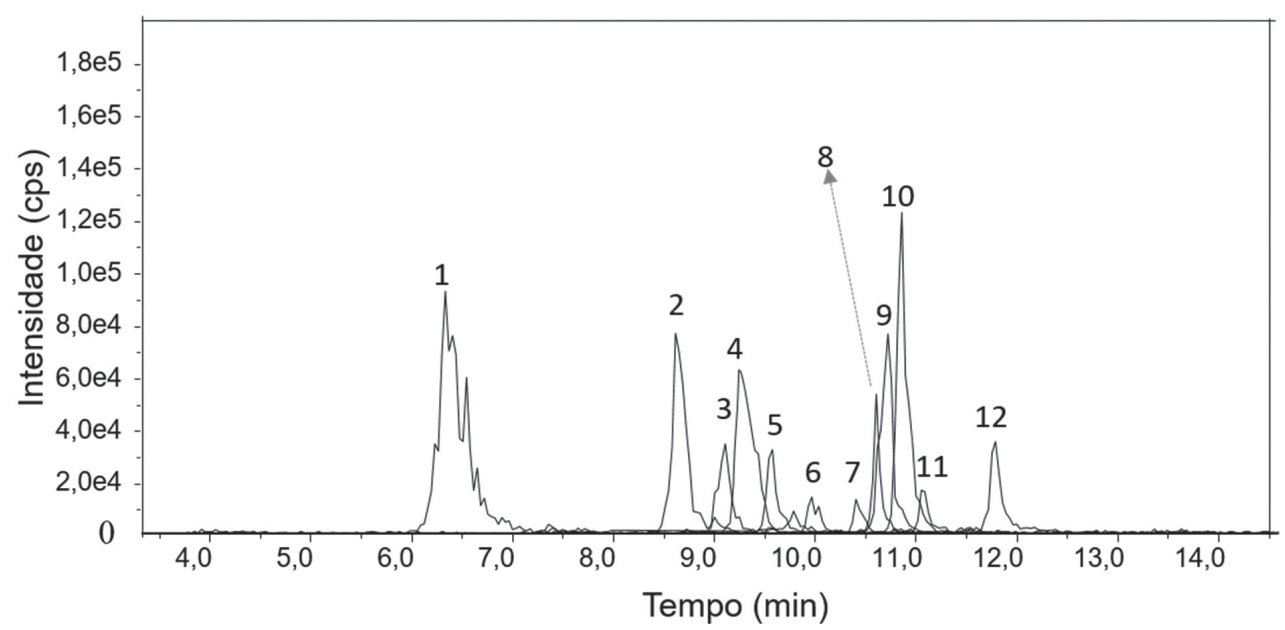

Figura 3. Cromatograma das análises de LC-ESI-MS/MS em extratos de folhas de amoreira preta. Numeração dos sinais: 1, ácido protocatecuico; 2 , ácido 4-hidroximetilbenzóico; 3, Ácido clorogênico; 4 ácido cafeico; 5, ácido vanílico; 6, ácido siríngico; 7, ácido p-cumárico; 8, ácido ferúlico; 9, umbeliferona; 10, quercetina; 11, ácido salicílico; 12, ácido elágico

Morus nigra. ${ }^{27,30,31}$ Em contrapartida, os ácidos 4-hidroximetilbenzoico, salicílico e elágico não foram mencionados para esta espécie em trabalho científico até o momento.

O composto umbeliferona (9), com concentração de $0,261 \pm 0,009 \mathrm{mg} \mathrm{g}^{-1}$, destacou-se por ser a terceira substância em maior concentração, sendo a primeira vez que a umbeliferona é relatada em folhas da espécie de Morus nigra L. Dugo et al., ${ }^{32}$ em seus estudos com extratos etanólicos de folhas de Morus alba obtidos por maceração utilizando sistema de HPLC acoplado a um espectrômetro de massas, também identificaram essa molécula. A umbeliferona é uma cumarina, e essa, por sua vez, é um metabólito fenólico, sintetizada principalmente por plantas, podendo fornecer defesa contra patógenos e atuando na regulação do estresse oxidativo e hormonal. ${ }^{33}$ Diversas ações biológicas são atribuídas a esses compostos, tais como atividade antifúngica, atenuação de danos renais e propriedades antioxidantes..$^{34,35}$

\section{Determinação de minerais nas folhas de Morus nigra}

As concentrações de minerais nas folhas de Morus nigra e os limites de detecção (LOD) e quantificação (LOQ) obtidos por ICPMS estão apresentados na Tabela 6. O LOD na ordem de $\mathrm{mg} \mathrm{kg}^{-1}$ foi calculado considerando 3 vezes o desvio padrão de dez leituras do branco da amostra dividido pelo valor da inclinação da curva. Os valores obtidos de LD variaram entre 0,03 e $2 \mathrm{mg} \mathrm{kg}^{-1}$, demonstrando que a sensibilidade do método proposto é adequada. Para os limites de quantificação (LOQ) os cálculos são definidos como 3,3 vezes o LOD. Os valores para o desvio padrão relativo (RSD) em todos os elementos apresentaram-se menores que $10 \%$, indicando boa precisão do método. A faixa linear foi de 1 a $200 \mu \mathrm{g} \mathrm{L}^{-1}$ para todos os elementos exceto para $\mathrm{K}$, determinado por fotometria de chama, neste caso o equipamento é calibrado com solução padrão de $100 \mathrm{mg} \mathrm{L}^{-1}$.

As determinações foram realizadas em triplicata e os resultados são expressos como média \pm desvio padrão. Entre os elementos microminerais determinados, cobre $(\mathrm{Cu})$, ferro $(\mathrm{Fe})$, zinco $(\mathrm{Zn})$, cobalto (Co), vanádio (V), níquel (Ni), alumínio (Al) e manganês $(\mathrm{Mn})$, as concentrações mais altas foram encontradas para e Fe com $580 \pm 1 \mathrm{mg} \mathrm{kg}^{-1}$. Já em relação aos nutrientes necessários a saúde em maior concentração, os macrominerais, potássio $(\mathrm{K})$ e magnésio $(\mathrm{Mg})$ e apresentaram concentrações de $28333 \pm 577$ e $692 \pm 22 \mathrm{mg} \mathrm{kg}^{-1}$, respectivamente, sendo o potássio o elemento predominante entre todos os elementos analisados.

Yigit et al., ${ }^{36}$ ao avaliarem a concentração mineral de folhas de Morus nigra coletadas em certa região da Turquia, usando um 
Tabela 6. Concentração de minerais em folhas de amoreira preta (média \pm desvio padrão) e limites de detecção (LOD) e quantificação (LOQ) obtidos por ICP-MS e fotometria de chama

\begin{tabular}{cccc}
\hline Elementos & $\begin{array}{c}\mathrm{mg} \mathrm{kg}^{-1} \mathrm{de} \\
\text { amostra seca }\end{array}$ & LOD $\left(\mathrm{mg} \mathrm{kg}^{-1}\right)$ & LOQ $\left(\mathrm{mg} \mathrm{kg}^{-1}\right)$ \\
\hline $\mathrm{Co}$ & $0,08 \pm 0,01$ & 0,03 & 0,1 \\
$\mathrm{Ni}$ & $0,44 \pm 0,04$ & 0,03 & 0,1 \\
$\mathrm{Al}$ & $77,55 \pm 7,12$ & 0,3 & 1,0 \\
$\mathrm{Mn}$ & $120 \pm 9$ & 2 & 6,0 \\
$\mathrm{Zn}$ & $22,94 \pm 0,13$ & 0,1 & 0,5 \\
$\mathrm{Cu}$ & $4,74 \pm 0,18$ & 0,1 & 0,2 \\
$\mathrm{Mg}$ & $692 \pm 22$ & 0,04 & 0,1 \\
$\mathrm{~V}$ & $0,79 \pm 0,05$ & 0,4 & 1 \\
$\mathrm{Fe}$ & $580 \pm 1$ & 0,4 & 1 \\
$\mathrm{~K}^{*}$ & $28333 \pm 577$ & 0,3 & 1 \\
\hline
\end{tabular}

*Determinação por fotometria de chama.

método de fluorescência por raios $\mathrm{X}$, constataram valores de potássio de 15902,1 \pm 213,0 ppm $\left(\mathrm{mg} \mathrm{kg}^{-1}\right)$. Entretanto, essas concentrações podem variar de acordo com diferentes de espécies, práticas agrícolas, região, condições de solo e clima. O potássio é indispensável ao organismo humano, pois participa do equilíbrio ácido-base, balanço hídrico, bomba sódio-potássio, age na conversão da glicose em glicogênio e na condução de impulsos nervosos. ${ }^{37}$

A concentração de magnésio apresentou $692 \pm 22 \mathrm{mg} \mathrm{kg}^{-1}$, superior ao reportado para folhas de Morus alba em um estudo de variação sazonal, onde foram relatadas concentrações de magnésio de 4,4 a 4,8 mg kg${ }^{-1}$ de matéria seca. ${ }^{38}$ A concentração observada neste estudo demonstra que os extratos de amoreira preta podem ser uma fonte abundante de magnésio. A recomendação de ingestão diária de magnésio é de 310 a $320 \mathrm{mg}$ para homens e 400 a $420 \mathrm{mg}$ para mulheres. ${ }^{39} \mathrm{O}$ consumo de aproximadamente $58 \mathrm{~g}$ ao dia de folhas de amoreira preta poderia suprir $10 \%$ da ingestão diária recomendada para magnésio.

Uma ocorrência bastante oportuna investigada nos extratos foliares de amoreira preta é a concentração de ferro ser bem maior que a concentração de manganês, pois estudos indicam que o ferro e o manganês competem pelos mesmos transportadores sistêmicos. Além disso, quando a concentração de manganês é superior, essa pode levar a um desequilíbrio homeostático de ferro no organismo. ${ }^{40}$ Investigações sobre a composição mineral de frutos de Morus nigra por espectrometria de absorção atômica revelaram concentrações de zinco e cobre de 32 e $4 \mathrm{mg} \mathrm{kg}^{-1}$ respectivamente, concentrações semelhantes aos avaliados neste estudo. ${ }^{41}$

\section{CONCLUSÃO}

De acordo com os resultados obtidos para o efeito das condições de extração de compostos fenólicos de folhas de amoreira preta, a temperatura de secagem apresentou forte impacto no conteúdo fenólico total, indicando que temperaturas mais baixas $\left(50^{\circ} \mathrm{C}\right) \mathrm{de}$ secagem em estufa podem preservar os compostos bioativos. A temperatura de infusão também exibiu grande influência na extração de compostos fenólicos, neste caso, temperaturas mais elevadas proporcionaram melhores extrações. Ao contrário, o tempo não teve influencia significativa na extração de compostos fenólicos. Altas concentrações de compostos fenólicos totais foram encontradas nos extratos foliares de Morus nigra, assim como para flavonóis e flavonoides. Além disso, os extratos exibiram boa capacidade antioxidante.
A análise por LC-ESI-MS/MS permitiu a identificação de 12 compostos de natureza fenólica nas folhas de amoreira preta, entre eles a quercetina, umbeliferona e ácido cafeico, que segundo a literatura são correlacionados à ação antioxidante. O composto umbeliferona, até o presente momento, não foi relatado em trabalhos científicos consultados em periódicos (Science Direct, Scopus, Google Acadêmico e outros), havendo relatos dessa substância na literatura apenas para as folhas de Morus alba L.

Por fim, as folhas de amoreira preta são ricas em micronutrientes minerais, sendo que dentre os 10 elementos determinados, potássio, ferro, manganês e magnésio apresentaram as maiores concentrações.

\section{AGRADECIMENTOS}

Os autores agradecem à Universidade Tecnológica Federal do Paraná, à Universidade Federal de Santa Catarina, à CAPES e ao CNPq pelo apoio financeiro.

\section{REFERÊNCIAS}

1. Wink, M. Em Encyclopedia of Evolutionary Biology; Wink, M., ed.; Richard M. Kliman, 2016.

2. Garbisa, S.; Sartor, L.; Biggin, S.; Salvato, B.; Benelli, R.; Albini, A.; Cancer 2001, 91, 822.

3. Russo, A.; Acquaviva, R.; Campisi, A.; Sorrenti, V.; Di Giacomo, C.; Virgata, G.; Barcellona, M. L.; Vanella, A.; Cell Biol. Toxicol. 2000, 16, 91.

4. Park, E.; Lee, S. M.; Lee, J. E.; Kim, K. H.; J. Funct. Foods 2013, 5, 178.

5. Wu, C. H.; Chen, C. C.; Ou, T. T.; Chyau, C. C.; Chang, Y. C.; Wang, C. J.; J. Funct. Foods 2013, 5, 1620.

6. Katsube, T.; Tsurunaga, Y.; Sugiyama, M.; Furuno, T.; Yamasaki, Y.; Food Chem. 2009, 113, 964.

7. Zeni, A. L. B.; Moreira, T. D.; Dalmargo, A. P.; Camargo, A.; Bini, L. A.; Simionatto, E. L.; Scharf, D. R.; An. Acad. Bras. Ciênc. 2017, 89, 2805.

8. Dalmagro, A. P.; Camargo, A.; Zeni, A. L. B.; Metab. Brain Dis. 2017, 32, 1963

9. Gernand, A. D.; Schulze, K. J.; Stewart, C. P.; West, Jr. K. P.; Christian, P.; Nat. Rev. Endocrinol. 2016, 12, 274.

10. Chemat, F.; Vian, M. A.; Cravotto, G.; Int. J. Mol. Sci. 2012, 13, 8615.

11. Singleton, V. L.; Orthofer, R.; Lamuela-Raventos, R. M.; Methods Enzymol. 1999, 299, 152

12. Lees, D. H.; Francis, F. J.; Hortscience 1972, 7, 83.

13. Yermakov, A. I.; Arasimov, V. V.; Yarosh, N. P.; Am. J. Plant Sci. 1987, $4,25$.

14. Brand-Williams, W.; Cuvelier, M. E.; Berset, C. L. W. T.; LWT Food Sci. Technol. 1995, 28, 25.

15. Boroski, M.; Aguiar, A. C.; Boeing, J. S.; Rotta, E. M.; Wibby, C. L.; Bonafé, E. G.; Souza, N. E.; Visentainer, J. V.; Food Chem. 2011, 125, 696.

16. Berker, K. I.; Ozdemir Olgun, F. A.; Ozyurt, D.; Demirata, B.; Apak, R.; J. Agric. Food Chem. 2013, 61, 4783.

17. Lane, J. H.; Eynon, L.; Normam Rodge 1934, 8.

18. Schulz, M.; Borges, G. S. C.; Gonzaga, L. V.; Seraglio, S. K. T.; Olivo, I. S.; Azevedo, M. S.; Nehring, P.; Gois, J. F.; Almeida, T. S.; Vitali, L.; Spudeit, D. A.; Micke, G. A.; Gallindo, D. L. B.; Fett, R.; Food Res. Int. 2015, 77, 125.

19. Ribani, M.; Bottoli, C. B. G.; Collins, C. H.; Jardim, I. C. S. F.; Melo, L. F. C.; Quim. Nova 2004, 27, 771.

20. Sánchez-Salcedo, E. M.; Mena, P.; García-Viguera, C.; Martínez, J. J.; Hernández, F.; J. Funct. Foods 2015, 18, 1039.

21. Vizzotto, M.; Pereira, M. C.; Rev. Bras. Frutic. 2011, 33, 1209. 
22. Ferreira, J. C.; Patino, C. M.; Jornal Brasileiro de Pneumologia 2015, $41,3713$.

23. Larrauri, J. A.; Ruperez, P.; Saura-Calixto, F.; J. Agric. Food Chem. 1997, 45, 1390.

24. Casagrande, M.; Zanela, J.; Wagner, A. J.; Busso, C.; Wouk, J.; Iurckevicz, G.; Mantanher, F.; Yamashita, F.; Malfatti, C. R. M.; Ind. Crops Prod. 2018, 125, 207.

25. Rajha, H. N.; El Darra, N.; Hobaika, Z.; Boussetta, N.; Vorobiev, E.; Maroun, R. G.; Louka, N.; Food Nutr. Sci. 2014, 5, 397.

26. Thabti, I.; Elfalleh, W.; Hannachi, H.; Ferchichi, A.; Campos, M. D. G.; J. Funct. Foods 2012, 4, 367.

27. Dalmagro, A. P.; Camargo, A.; Silva Filho, H. H.; Valcanaia, M. M.; Jesus, P. C.; Zeni, A. L. B.; Ind. Crops Prod. 2018, 123, 323.

28. Volpato, G. T.; Calderon, I. M. P.; Sinzato, S.; Rudje, M. V.; Damasceno, D. C.; J. Ethnopharmacol. 2011, 138, 691.

29. Enkhmaa, B.; Shiwaku, K.; Katsube, T.; Kitajima, K.; Anuurad, E.; Yamasaki, M.; J. Nutr. 2005, 135, 729.

30. Radojković, M.; Zekovic, Z.; Mašković, P.; Vidović, S.; Mandić, A; Mišan, A; Durović, S.; J. Supercrit. Fluids 2016, 117, 50.

31. Sánchez-Salcedo, E. M.; Mena, P.; García-Viguera, C.; Martínez, J. J.; Hernández, F.; J. Funct. Foods 2015, 12, 399.
32. Dugo, P.; Donato, P.; Cacciola, F.; Germanò, M. P.; Rapisarda, A.; Mondello, L.; J. Sep. Sci. 2009, 32, 3627.

33. Bourgaud, F.; Hehn, A.; Larbat, R.; Doerper, Gontier, E.; Kellner, S.; Matern, U.; Phytochem. Rev. 2006, 5, 293.

34. Pan, L.; Li, X.; Yan, Z.; Guo, H.; Qin, B.; Plant Physiol. Biochem. 2015, 97, 272.

35. Garud, M. S.; Kulkarni, Y. A.; Pharmacol. Rep. 2017, 69, 1263.

36. Yigit, D.; Akar, F.; Baydas, E.; Buyukyildiz, M. Asian J. Chem. 2010, 22, 3554.

37. He, F. J.; Macgregor, G. A.; Br. Med. J. 2001, 323, 497.

38. Vento, B.; Martínez Carretero, E.; Moreno, G.; Duplancic, A.; Bot. Lett. 2017, 162, 183 .

39. Food and Nutrition Board, Institute of Medicine, Dietary Reference Intakes for Calcium, Phosphorus, Magnesium, Vitamin D and Fluoride. National Academy Press: Washington DC, 1997.

40. Fitsanakis, V. A.; Zhang, N.; Garcia S.; Aschner, M.; Neurotoxic. Res. 2010, 18, 124.

41. Ercisli, S.; Orhan, E.; Food Chem. 2007, 103, 1380. 